\title{
Volume Preserving Image Registration
}

\author{
Eldad Haber ${ }^{1}$ and Jan Modersitzki ${ }^{2 \star}$ \\ 1 Dept. of Mathematics and Computer Science, Emory University, Atlanta GA \\ 30322, haber@mathcs . emory . edu \\ 2 Inst. of Mathematics, University of Lübeck, Germany \\ modersitzki@math.uni-luebeck.de
}

\begin{abstract}
In this paper we discuss image registration techniques with a focus on volume preserving constraints. These constraints can reduce the non-uniqueness of the registration problem significantly. Our implementation is based on a constrained optimization formulation. To solve the problem we use a variant of the Sequential Quadratic Programming method. Moreover, we present results on synthetic as well as on real-life data.
\end{abstract}

\section{Introduction}

Image registration is one of the fundamental tasks in today's image processing and in particular in medical imaging; see, e.g., [1,2 and references therein. The objective of image registration is to make images which are taken at different times, from different perspectives, and/or from different devices to be more alike. Loosely, the goal of image registration is to find a "reasonable" deformation such that the "distance" between a reference image $R$ and a deformed version of a template image $T$ becomes small.

An application of particular clinical interest is the registration of pairs of images acquired before and after contrast administration; see, e.g., [3] and references therein. A typical example is depicted in Fig. 1. In this application, magnetic resonance images of a female breast are taken at different times (images from Bruce Daniel, Lucas Center for Magnetic Resonance Spectroscopy and Imaging, Stanford University). The first image shows an MRI section taken during the so-called wash-in phase of a radiopaque marker and the second image shows the analogous section during the so-called wash-out phase. A comparison of these two images indicates a suspicious region in the upper part of the images. This region can be detected easily if the images have been registered: tissue located at a certain position in the wash-in image is related to the tissue at the same position in the wash-out phase. Generally, however, a quantitative analysis is a delicate matter since observable differences are not only related to contrast uptake but also due to motion of the patient, like, for example, breathing or heart beat.

\footnotetext{
* Jan Modersitzki was supported by the US National Institutes of Health under Grant NIHR01 HL 068904.
} 
As pointed out by Rohlfing et al. 3, there is a substantial difficulty with the registration of pre and post-contrast images. Bright regions seem to enlarge during the so-called wash-in phase. This enhancement is due to contrast uptake but not to movement of the patient. Fig. 3 illustrates an ideal situation. Without external information, it is impossible to answer whether the white area has been enlarged or the grey area turned to white.

In this paper, we present a flexible constrained image registration approach. It has three main ingredients: a distance measure, a regularizer, and the constraints. Our framework is general enough to handle a variety of distance measures, including the most popular ones, like those based on the sum of squared differences (SSD) (cf., e.g., 4]), mutual information (MI) (cf., e.g., [5]), or correlation, as long as a Gâteaux derivative exists; see, e.g., 78]. For presentation purposes, we explicitly discuss the approach only for the SSD measure.
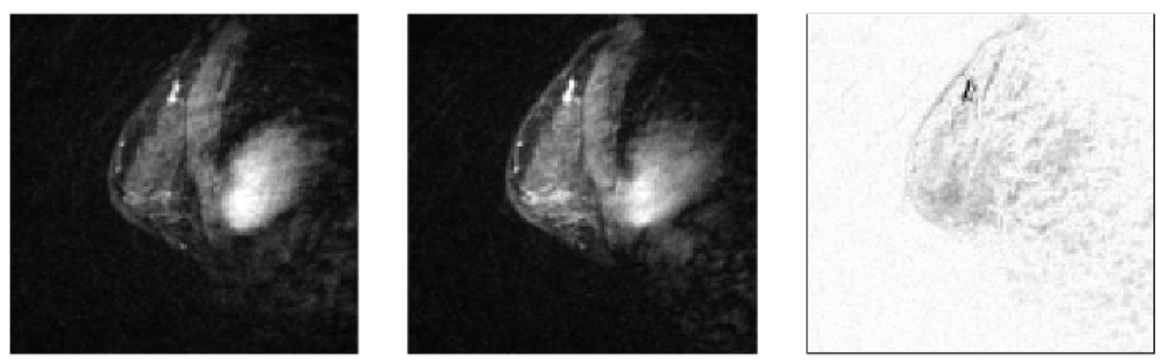

Fig. 1. MRI's of a female breast, LEFT: during the wash-in phase, MIDDLE: during the wash-out phase, and RIGHT: difference image.

\section{Mathematical Setup and Discretization}

With $d \in \mathbb{N}$ we denote the spatial dimension of the given images $R, T: \mathbb{R}^{d} \rightarrow \mathbb{R}$. Thus, $T(\boldsymbol{x})$ gives a gray value at a spatial position $\boldsymbol{x}$. We assume that the supports of the images are contained in a bounded domain $\Omega:=] 0, L{ }^{d}$, i.e. $R(\boldsymbol{x})=T(\boldsymbol{x})=0$ for $\boldsymbol{x} \notin \Omega$.

Our goal is to find a "reasonable" deformation $\boldsymbol{u}$ such that the "distance" between the reference image $R$ and the deformed template image $T(\boldsymbol{x}+\boldsymbol{u}(\boldsymbol{x}))$ becomes small. It is well-known that this problem is ill-posed and therefore needs to be regularized, see, e.g., 9[10]. A formulation of the (VP) constrained problem thus reads

$$
\begin{aligned}
\operatorname{minimize} & \mathcal{D}[R, T ; \boldsymbol{u}]+\alpha \mathcal{S}[\boldsymbol{u}] \\
\text { subject to } & \mathcal{C}[\boldsymbol{u}](\boldsymbol{x}):=\operatorname{det}\left(I_{d} \nabla \boldsymbol{u}(\boldsymbol{x})\right)-1=0 \quad \text { for all } \quad \boldsymbol{x} \in \Omega,
\end{aligned}
$$

where $\mathcal{D}$ is some distance measure (e.g. the sum of squared difference) and $\mathcal{S}$ is some regularization term (e.g. the elastic regularizer). Here, $\alpha>0$ is a regularization parameter and compromises between similarity and regularity. For 
ease of presentation, we assume that $\mathcal{S}$ is defined via a bilinear form, at it is the case for popular regularizer, like, e.g., the elastic [11213], fluid [1214, diffusion [15], or curvature regularizers [16].

Choosing a stable discretization method for an optimization problem with a differential constraint is a delicate matter. Similar to [17 18], we use staggered grids. Though staggered grids seem to be natural for the discretization of the registration problem on a regular grid, we are not aware of any registration scheme where this discretization is used.
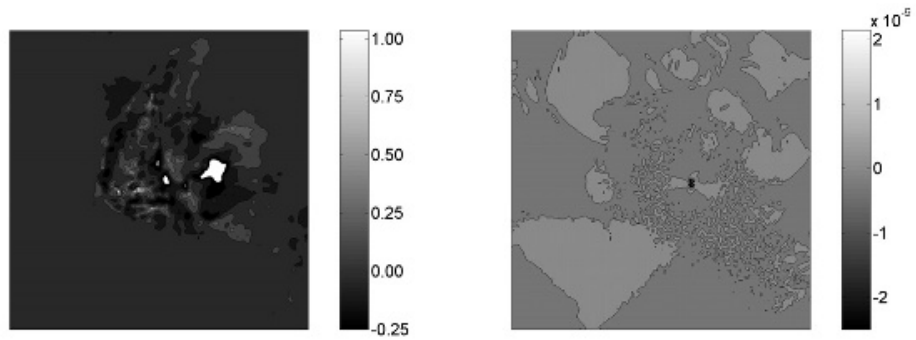

Fig. 2. Volume preservation of the unconstrained (LEFT) and constrained (RIGHT) registration results for the MRI example.

\section{Solving the Discrete Optimization Problem}

Let $\boldsymbol{U}, \boldsymbol{D}, \boldsymbol{S}$, and $\boldsymbol{C}$ denote the discrete analogs of $\boldsymbol{u}, \mathcal{D}, \mathcal{S}$, and $\mathcal{C}$. The discrete analog of the image registration problem (1) is phrased as follows,

$$
\begin{array}{ll}
\text { minimize } & \boldsymbol{D}(\boldsymbol{U})+\alpha \boldsymbol{S}(\boldsymbol{U}) \\
\text { subject to } & \boldsymbol{C}(\boldsymbol{U})=0 .
\end{array}
$$

In order to solve problem (21) numerically we use the framework of Sequential Quadratic Programming (SQP); see [19] for a detailed discussion. With the Lagrange multiplier $\boldsymbol{P}$, the Lagrangian of the problem is

$$
\boldsymbol{L}(\boldsymbol{U}, \boldsymbol{P})=\boldsymbol{D}(\boldsymbol{U})+\alpha \boldsymbol{S}(\boldsymbol{U})+\boldsymbol{C}(\boldsymbol{U})^{\top} \boldsymbol{P} .
$$

Differentiating with respect to $\boldsymbol{U}$ and $\boldsymbol{P}$, we obtain the Euler-Lagrange equations

$$
\begin{aligned}
& 0=\boldsymbol{L}_{\boldsymbol{U}}(\boldsymbol{U}, \boldsymbol{P})=\boldsymbol{D}_{\boldsymbol{U}}(\boldsymbol{U})+\alpha \boldsymbol{S}_{\boldsymbol{U}}(\boldsymbol{U})+\boldsymbol{C}_{\boldsymbol{U}}(\boldsymbol{U})^{\top} \boldsymbol{P}, \\
& 0=\boldsymbol{L}_{\boldsymbol{P}}(\boldsymbol{U}, \boldsymbol{P})=\boldsymbol{C}(\boldsymbol{U}) .
\end{aligned}
$$

We can now solve the nonlinear system (3) numerically by using a Newton-type method; see [18] for details. 

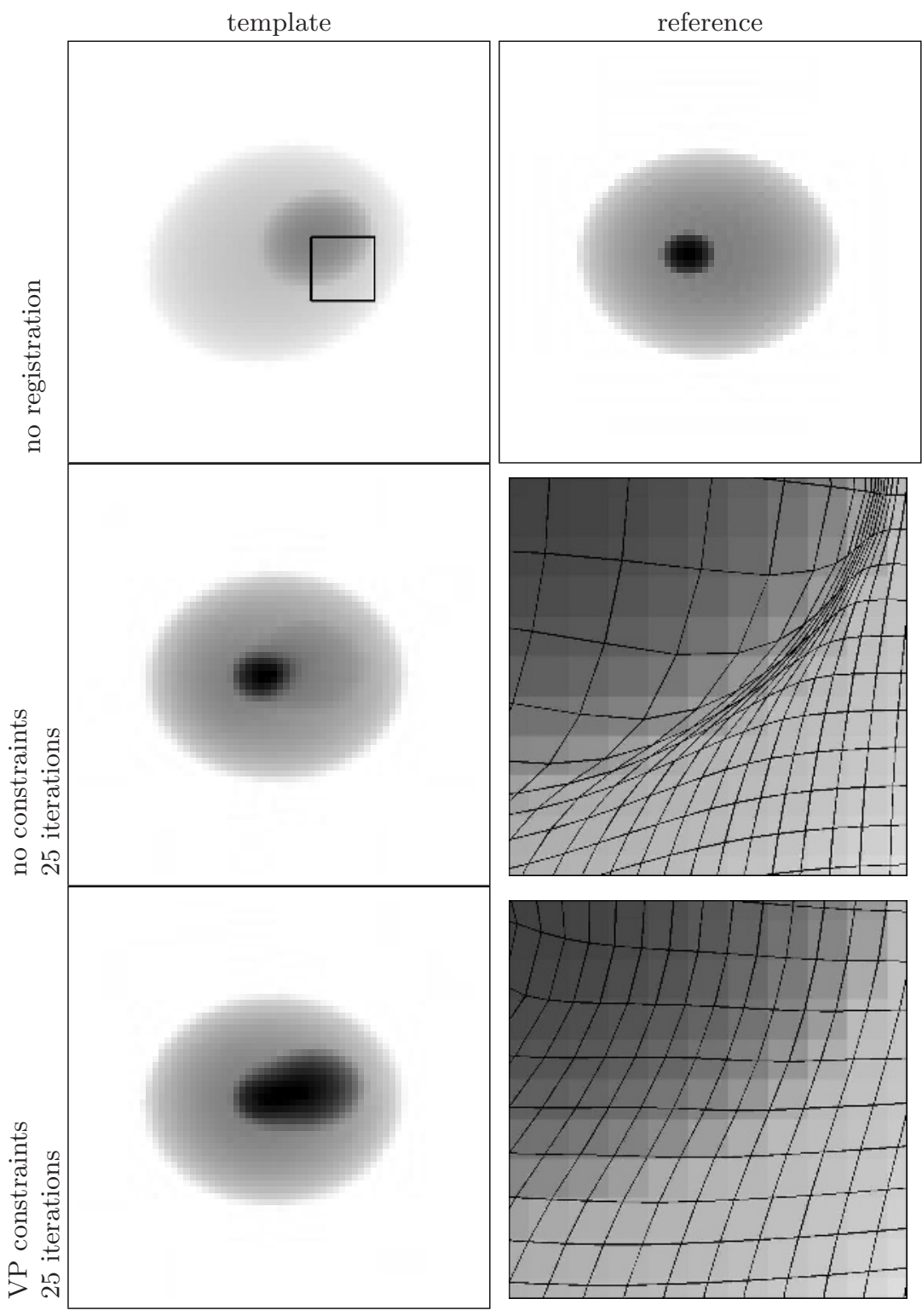

Fig. 3. Synthetic example, LEFT COLUMn: deformed template, RIGHT COLUMN: reference and detail of the deformed grid; TOP ROW: template and reference, no registration, MIDDLE ROW: deformed template and details with grid after unconstrained registration, воттом Rоw: deformed template and details with grid after VP constrained registration. For both schemes, we choose $\alpha=10^{3}$ and stopped after 25 iterations. 
deformed $T$
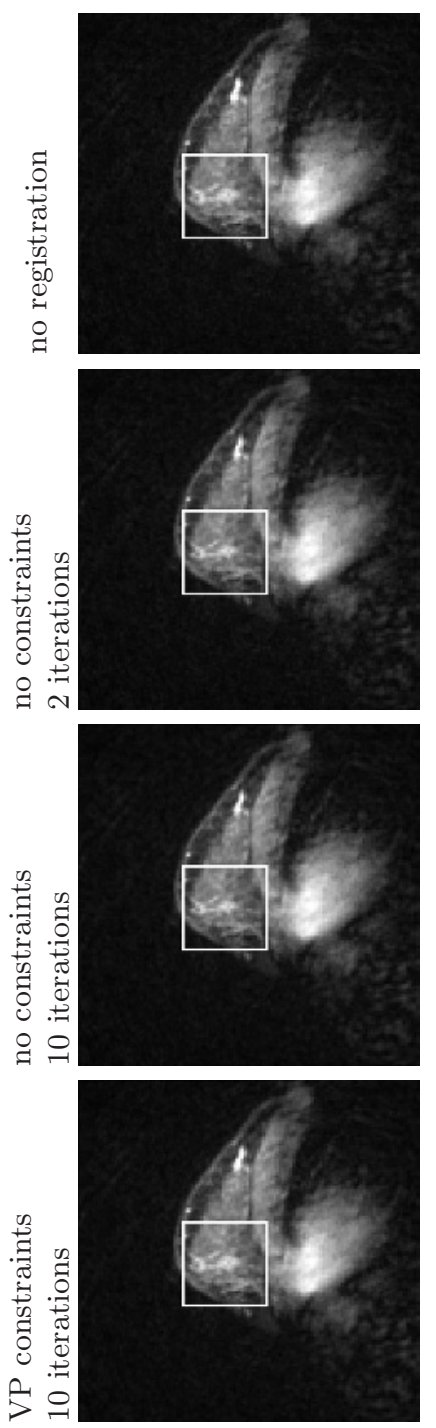

difference
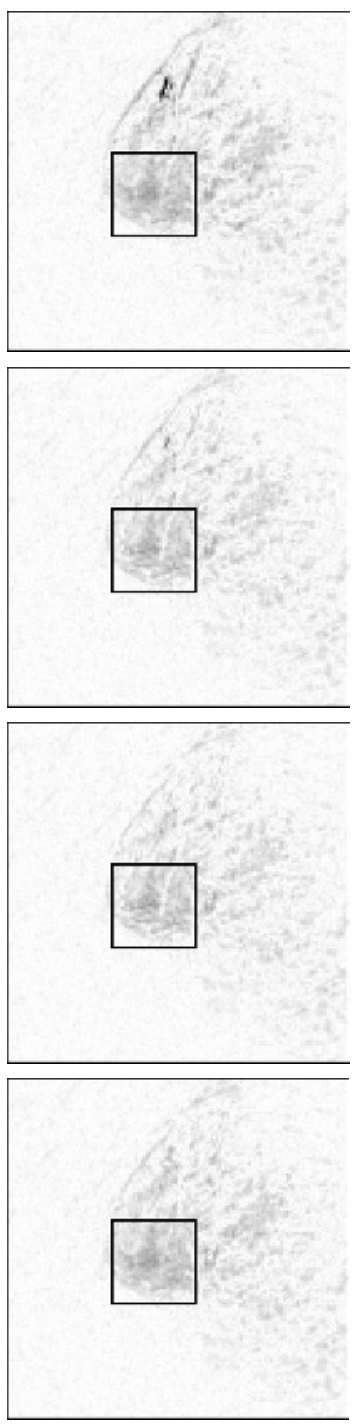

difference with nodal grid
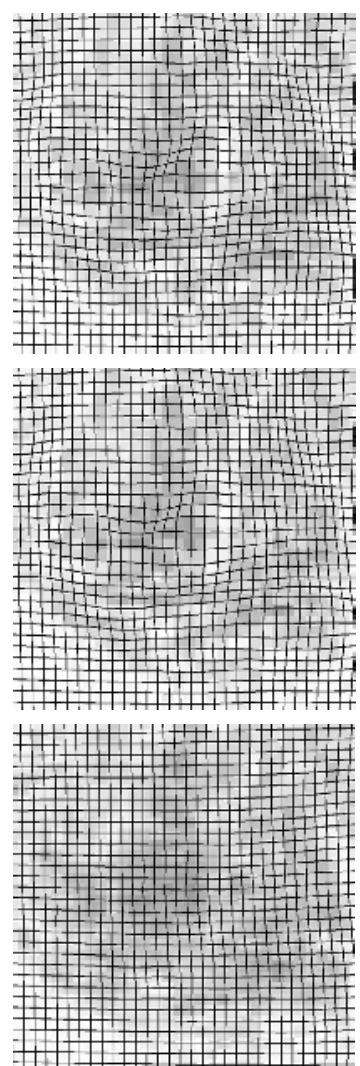

Fig. 4. Registration results for the images of Fig. 1. LEFT COLUMN deformed template images $T_{u}$, MIDDLE COLUMN difference image $\left|R-T_{u}\right|$ with region of interest (ROI), RIGHT COLUMN ROI with nodal grid, vertices connected by straight lines ; ROW 1: no registration, ROW 2: no constraints two iterations, ROW 3: no constraints ten iterations, and ROW 4: volume preserving constraints ten iterations.

\section{Numerical Examples}

The Blob: To illustrate the potential of the volume preserving registration we present a synthetic example; see Fig. 3. The reference image (top right) shows 
an elliptic global structure which contains a small almost circular object. The template (top left) shows a rotated version of the global ellipse, where the inner structure is translated and considerably enlarged. Note that this example mimics the situation for contrast enhanced images: slightly deformed global structures, where inner structures may change drastically due to contrast uptake.

As it is apparent from Fig. 3 the unconstrained registration gives very good results if we are looking at the difference between the reference and deformed template images alone. However, as expected, the inner structure has been re-

Table 1. Computation time (using MATLAB 6.5 on a DELL Inspiron 8600 Notebook) and iterations for the unconstraint and VP constraint registrations. The stopping criteria is $\left\|\boldsymbol{u}^{\text {old }}-\boldsymbol{u}\right\| \leq \operatorname{tol}_{U}:=10^{-1}$.

\begin{tabular}{|r|r|r|}
\hline image size & unconstraint & VP constrained \\
\hline $64 \times 64$ & $28 \mathrm{~s}, \# 38$ & $69, \# 21$ \\
$128 \times 128$ & $296 \mathrm{~s}, \# 68$ & $846, \# 23$ \\
\hline
\end{tabular}

Table 2. Numerical results for the un- and VP-contrained registrations; $k$ is the number of iteration performed.

\begin{tabular}{|c|ccc|}
\hline & $k$ & $\boldsymbol{D}\left(\boldsymbol{U}^{(k)}\right) / \boldsymbol{D}(0)$ & $\| \boldsymbol{C}\left(\boldsymbol{U}^{(k)} \|_{\infty}\right.$ \\
\hline blob unconstrained & 25 & 0.21 & 0.87 \\
VP constrained & 25 & 0.73 & $\leq 10^{-6}$ \\
\hline MRI unconstrained & 2 & 0.81 & 1.36 \\
unconstrained & 10 & 0.78 & 1.36 \\
VP constrained & 10 & 0.87 & $\leq 10^{-6}$ \\
\hline
\end{tabular}

duced so as to fit the one in the reference image. This results in a drastic change of volume, which can be observe from the visualization of a part of the grid in Fig. 3 (middle right) corresponding to a region of interest emphasized in the template image (top left). Thus, for contrast enhanced images, the registration gives meaningless results, though the difference is small.

Fig. 3 also shows the results of the volume preserving registration (bottom left). As is apparent from this figure, the global deformation has been resolved, the inner ellipse has been moved to match the inner ellipse in the reference image. However, the volume of the inner ellipse has not been altered, which leads to a larger difference as in the unconstrainted case but also to a more realistic registration; see also the deformed grid (bottom right). Computation time and numerical values for the difference $\boldsymbol{D}$ and the constraints $\boldsymbol{C}$ for the un- and VP-constrained registration are summarized in Table 1 and 2.

MRI scans: In our second example, we discuss results obtained for the images shown in Fig. 1. Fig. 4 shows the results after two ( 2nd row) and ten iterations (3rd row) of the unconstrained registration as well as after ten iterations of the VP constrained registration (4th row). After at most ten iteration both schemes have converged. 
Although the numbers (cf. Table 2) indicate a larger reduction of the difference by the unconstrained registrations, the ranking is not so clear if one looks at the difference images, cf. Fig. 4 Here, the difference after ten steps un- and VP constrained registration looks pretty much the same. After two steps of the unconstrained registration the bright spot in the top part of the image has not been resolved satisfiably. The explanation is that small spots which are related to noise in the MRI images and hardly visible in the images are registered in the unconstrained registration. This leads to a large reduction though it is hardly visible. To remove this small spots, the volume has to be changed locally. However, the registration of these small spots does not contribute to a meaningful solution for this problem.

In Fig. 2 we display the pointwise map of the change of volume. Using the unconstrained approach, we observe a considerable change of volume for the breast with a peak value of 1.36. Thus, part of the breast has been enlarged by a factor of 2.36. For the constrained approach, we observe that the volume change is below a user supplied threshold (here, $\mathrm{tol}_{C}=1 o^{-6}$ ) everywhere. In fact, since we used a quasi-Newton scheme for projection, the numbers are around $10^{-9}$.

\section{References}

1. Maurer, C.R., Fitzpatrick, J.M.: A Review of Medical Image Registration. In: Interactive Image-Guided Neurosurgery. Park Ridge, IL, American Association of Neurological Surgeons (1993) 17-44

2. Fitzpatrick, J.M., Hill, D.L.G., Jr., C.R.M.: Image registration. In Sonka, M., Fitzpatrick, J.M., eds.: Handbook of Medical Imaging, Volume 2: Medical Image Processing and Analysis, SPIE (2000) 447-513

3. Rohlfing, T., Maurer, Jr., C.R., Bluemke, D.A., Jacobs, M.A.: Volume-preserving nonrigid registration of MR breast images using free-form deformation with an incompressibility constraint. IEEE Transactions on Medical Imaging 22 (2003) 730-741

4. Brown, L.G.: A survey of image registration techniques. ACM Computing Surveys 24 (1992) 325-376

5. Collignon, A., Vandermeulen, A., Suetens, P., Marchal, G.: 3d multi-modality medical image registration based on information theory. Kluwer Academic Publishers: Computational Imaging and Vision 3 (1995) 263-274

6. Viola, P., Wells III, W.M.: Alignment by maximization of mutual information. (1995) 16-23 IEEE 1995.

7. Roche, A.: Recalage d'images médicales par inférence statistique. PhD thesis, Université de Nice, Sophia-Antipolis, France (2001)

8. Hermosillo, G.: Variational methods for multimodal image matching. PhD thesis, Université de Nice, France (2002)

9. Clarenz, U., Droske, M., Rumpf, M.: Towards fast non-rigid registration. In: Inverse Problems, Image Analysis and Medical Imaging, AMS Special Session Interaction of Inverse Problems and Image Analysis. Volume 313., AMS (2002) 67-84

10. Modersitzki, J.: Numerical Methods for Image Registration. Oxford University Press (2004)

11. Broit, C.: Optimal Registration of Deformed Images. PhD thesis, Computer and Information Science, University of Pensylvania (1981) 
12. Christensen, G.E.: Deformable Shape Models for Anatomy. PhD thesis, Sever Institute of Technology, Washington University (1994)

13. Fischer, B., Modersitzki, J.: Fast inversion of matrices arising in image processing. Num. Algo. 22 (1999) 1-11

14. Bro-Nielsen, M.: Medical Image Registration and Surgery Simulation. PhD thesis, IMM, Technical University of Denmark (1996)

15. Fischer, B., Modersitzki, J.: Fast diffusion registration. AMS Contemporary Mathematics, Inverse Problems, Image Analysis, and Medical Imaging 313 (2002) 117129

16. Fischer, B., Modersitzki, J.: Curvature based image registration. J. of Mathematical Imaging and Vision 18 (2003) 81-85

17. Yee, K.: Numerical solution of initial boundary value problems involving Maxwell's equations in isotropic media. IEEE Trans. on Antennas and Propagation 14 (1966) 302-307

18. Haber, E., Modersitzki, J.: Numerical methods for volume preserving image registration. Technical Report TR-2004-012-A, Department of Mathematics and Computer Science, Emory University, Atlanta GA 30322 (2004) Submitted to Inverse Problems.

19. Nocedal, J., Wright, S.: Numerical optimization. Springer, New York (1999) 